\title{
La Société huronne
}

\section{Lucien Campeau}

Volume 50, numéro 1, 1983

Bilan de l'histoire religieuse au Canada

Canadian Catholic History: A survey

URI : https://id.erudit.org/iderudit/1007049ar

DOI : https://doi.org/10.7202/1007049ar

Aller au sommaire du numéro

Éditeur(s)

Les Éditions Historia Ecclesiæ Catholicæ Canadensis Inc.

ISSN

0318-6172 (imprimé)

1927-7067 (numérique)

Découvrir la revue

Citer cet article

Campeau, L. (1983). La Société huronne. Sessions d'étude - Société canadienne d'histoire de l'Église catholique, 50(1), 325-339.

https://doi.org/10.7202/1007049ar

Tous droits réservés @ Les Éditions Historia Ecclesiæ Catholicæ Canadensis Inc., 1983
Ce document est protégé par la loi sur le droit d'auteur. L'utilisation des services d'Érudit (y compris la reproduction) est assujettie à sa politique d'utilisation que vous pouvez consulter en ligne.

https://apropos.erudit.org/fr/usagers/politique-dutilisation/ 


\section{La Société huronne}

Je voudrais, dans le présent exposé, tracer une esquisse de la société huronne telle qu'elle apparaît dans les Relations de leurs missionnaires, publiées annuellement ou à peu près de 1635 à 1650 . Tout d'abord, il faudrait souligner la qualité de cette information. À mes yeux, elle a une valeur unique. D'abord, parce qu'elle raconte une expérience directe, vécue et racontée au jour le jour durant seize ans par ceux-là mêmes qui la font. Ensuite, parce que la société qui est objet d'observation est isolée, à plusieurs centaines de lieues des Français, demeurant tout ce temps à l'abri d'influences étrangères et transformantes. Les témoins, les missionnaires, bien qu'Européens, sont aussi les mieux accrédités à nous fournir un tel témoignage. Les Indiens se contentaient eux-mêmes de vivre. Leur culture ne leur fournissait pas ce regard réflexif qui est la source d'un témoignage cohérent et organisé. Les missionnaires, formés dans une culture millénairement habituée à un tel genre d'exercice intellectuel, fondé sur l'analyse, sur la comparaison et la synthèse, avaient cette facilité d'organiser un témoignage intelligible. La connaissance des langues, la communauté de vie, l'effort obligatoire d'adaptation, tout cela faisait d'eux des témoins par excellence. Certes, ils étaient des Européens et ils avaient les préjugés propres à leur culture. Mais c'est le rôle de l'historien de dissocier le témoignage du témoin et de soupeser ce qui en demeure scientifiquement valable. Les Indiens n'eussent pu exprimer seulement le témoignage. Et nul autre que les missionnaires, ni les fonctionnaires connaissant les autochtones de loin et par ouïdire, ni les voyageurs, témoins en passant et sans expérience quotidienne, n'auraient pu présenter cette information abondante, vécue, souvent revue et corrigée, que l'on trouve dans les Relations des Hurons. Voilà où nous puisons les connaissances que nous exposons, sans négliger Champlain et Sagard qui les ont précédés ${ }^{1}$.

\footnotetext{
${ }^{1}$ Ce texte est un résumé et un extrait d'une étude beaucoup plus étendue et détaillée qui est en cours. Les sources sont celles que nous indiquons dans le texte et que le lecteur n'aura pas de peine à retracer. C'est pourquoi nous nous abstenons de faire des références précises, qu'il aurait fallu multiplier et même expliquer. À l'occasion seulement, nous préciserons les titres d'autres ouvrages mentionnés en passant.
} 
Je n'ai pas à vous apprendre que les autochtones venus en contact avec les Européens en Amérique du Nord durant la période française appartenaient à deux groupes linguistiques différents: les Algonquiens et les Iroquiens ${ }^{2}$. Sur le plan culturel, il n'y a pas entre eux de césure bien marquée, bien que les deux se distribuent selon une gamme continue, qui va du pur nomade au proto-agriculteur sédentaire et villageois. Chez les Algonquiens surtout, on perçoit des étapes qui vont de la chasse nomade à l'agriculture sédentaire. Les Iroquiens semblent tous avoir été des proto-agriculteurs, la sédentarité étant l'un des premiers effets de l'occupation agricole. Les témoins européens, habitués aux sociétés politiques complexes de leur continent, en ont perçu de semblables sur le nouveau, parlant d'Abénaquis, de Souriquois, d'Algonquins, d'Iroquois et de Hurons. Il est nécessaire, croyons-nous, de mettre le doigt sur la communauté socio-politique indigène, si l'on veut parler avec compétence de ses institutions. Chez les nomades, cette communauté est la bande, occupant un territoire déterminé. Elle gère ses intérêts collectifs d'une manière indépendante et autonome. Le nord du Québec et les Maritimes étaient peuplés par ces bandes dissociées, parfois même en guerre les unes contre les autres en dépit de la communauté de culture. Chez les Iroquois et les Hurons, la communauté socio-politique se trouvait au niveau de ce que les missionnaires appelaient la nation: il y en avait quatre chez les Hurons et cinq chez les Iroquois. Les Maskoutens, formation algonguienne vivant à l'ouest de Détroit, étaient aussi composés de plusieurs nations.

Au temps où arrivent les Français, un trait général des sociétés iroquiennes était d'être groupées en plusieurs ligues. Les mieux connues sont les ligues iroquoise et huronne. Mais il y en avait d'autres. Voisine des Hurons était la ligue des Pétuns, composée de deux nations. La plus puissante et la plus nombreuse était celle des Neutres, répandue sur les rives du Lac Érié et comptant sept nations. C'est le nombre et la puissance des Neutres, plutôt que leur habileté politique, qui les maintenait en paix à la fois avec les Iroquois et les Hurons, ennemis irréconciliables. Les Tuscaroras, à l'extrémité sud du monde iroquien, formaient aussi une ligue. On présume que les Andastes et les Ériés en avaient de semblables, bien que manquent les témoignages explicites. En tout cas, les Maskoutens, ennemis et voisins des Neutres, étaient groupés de même façon, bien qu'ils fussent algonquiens. La formule de la ligue n'était probablement pas très ancienne. La ligue iroquoise ne semble pas

\footnotetext{
${ }^{2}$ Nous nous excusons d'adopter cette forme, plutôt qu'iroquoien. Mais ce dernier nous paraît d'assonnance difficile et peu conforme au génie français.
} 
remonter plus loin que la fin du seizième siècle, la tradition en attribuant la fondation à un prophète huron. Et il est vraisemblable que les Hurons aient été les inventeurs de ce genre d'alliances pemanentes. Le P. Jérôme Lalemant, en effet, faisait remonter à plus de deux cents ans, c'està-dire avant 1440 , l'union des deux plus vieilles nations huronnes, les Attignaouantans et les Attingnenongnahacs. Deux autres s'étaient ajoutées à elles, l'une vers 1590 et l'autre vers 1610. Les Attignaouantans, la nation la plus nombreuse et occupant le territoire le plus étendu et le plus favorable, paraissent avoir été le berceau de la ligue. Leur distribution en petits villages souvent non fortitiés, à la différence des autres nations iroquiennes, leur confere aussi un caractère particulier d'antiquité. On devine que la formation des ligues a eu lieu en un temps d'urgences pressantes. Car à celui de la mission jésuite et après, les différentes ligues n'acceptaient plus de nouvelles nations à titre de constituantes des ligues. On se contentait alors d'adopter les groupes adventices, sans leur donner de territoire particulier ou une voix propre au conseil de ligue, Les Ouenrohronons furent ainsi reçu par les nations huronnes en 1638. Et les Tohontaenrats, les Ahrendaronons et les Tuscaroras furent adoptés des Tsonnontouans par la suite, sans être membres de la ligue iroquoise.

Le but essentiel de la ligue est de procurer un forum où régler les conflits entre nations qui aboutiraient à verser mutuellement leur sang. Un tel organisme existe dans la nation elle-même, sous forme de conseil érigé en tribunal. Mais entre nations désunies, le sang versé provoque inévitablement à rendre la pareille à la nation coupable. Ainsi s'enchaînent les vendettas interminables que sont les guerres indiennes. La ligue procure un tribunal où une nation contractante peut et doit réclamer satisfaction contre une autre. De la sorte la paix est conservée entre les membres de la ligue. Pour le reste des problèmes communs, il appartient à chacune des composantes, ordinairement jalouse de son initiative, de les proposer à la ligue et d'accepter ou de rejeter ses avis. La vie intime de chaque nation, surtout, échappe entièrement au conseil de la ligue.

La ligue n'est pas une communauté socio-politique. C'est à la nation, occupant un territoire propre et bien délimité, conservant une pleine autonomie de sa vie interne et de sa politique extérieure, qu'appartient ce caractère, à l'exception seulement de ce qu'elle veut bien concéder à l'intérêt commun de la ligue. Les nations d'une même ligue se prêtaient mutuellement secours en cas de nécessité, mais cela même manquait parfois. Les nations iroquoises avaient chacune leurs guerres, leurs ennemis et leurs alliés propres. Les huronnes, plus rap- 
prochées géographiquement les unes des autres, combattaient le plus souvent de concert. L'une d'elles, en chaque ligue, possédait une sorte de droit d'aînesse: les Onontagués chez les Iroquois; les Attignaouantans chez les Hurons.

Les rapports sociaux et politiques ont leur direction commune au sein de la nation. Celle-ci, en principe, est une lignée biologique. Les nations, écrit le P. Jérôme Lalemant, "ne sont presque distinguées que par diverses sources d'ayeuls et bisayeuls dont ils conservent chèrement les noms et la mémoire» ${ }^{3}$. Elles sont donc des descendances. Et des descendances masculines, non féminines, sans quoi un Français du dixseptième siècle n'aurait pu manquer de signaler la différence. Nous aurons à revenir sur le caractère masculin des lignées et des successions. En principe, donc, une nation est une lignée, descendue d'un aïeul; mais en fait, la nation huronne est un mélange de lignées. C'est qu'au cours de l'histoire, la lignée originelle en a adopté d'autres en son sein, qu'elle a intégrées à son appareil social, mais en laissant à chacune son identité et la garde de quelques traditions et intérêts particuliers. Les missionnaires ont encore donné le nom de nations à ces lignées adoptées, mais elles ne sont pas comptées comme composantes de la ligue. Une source de 1648 indique qu'il y avait quatre nations adoptées chez les Hurons ${ }^{4}$. Une telle lignée subalterne peut être d'ailleurs distribuée entre plusieurs nations, conservant son identité en chacune. On en possède une exemple historique dans les Ouenrohronons, adoptés en 1638. Ils se sont partagés entre les bourgs d'au moins trois nations huronnes.

Les Relations des Hurons sont constantes, de 1635 à 1650, à ne compter que quatre nations dans la ligue huronne. Ce sont les Attignaouantans, les Attingnenongnahacs, les Tohontaenrats et les Ahrendaronons. M. Heidenreich, dans son livre d'ailleurs remarquable ${ }^{5}$, a cru devoir en compter une cinquième, les Ataronchronons. Mais dans le volume même qui semble étayer sa thèse, le $P$. Jérôme Lalemant, en 1640, réaffirmait très explicitement et à deux reprises le nombre de quatre nations composantes. Cela est confirmé du fait qu'en 1637, on envoyait au principal chef des Ataronchronons la meilleure partie de la dépouille d'un Iroquois torturé, échu en propre à la nation des Attignaouantans. Les Ataronchronons ne peuvent être qu'une région des Attignaouantans,

3 R.G. Thwaites, The Jesuit Relations and Allied Documents, 73 vol., Cleveland, 1896-1901, t. 6, p. 226.

${ }^{4}$ Ibid., pp. 33 et 242.

${ }^{5}$ Conrad Heidenreich, Huronia, pp. 84-5. 
comprenant le littoral entier de la baie de Matchedash et occupée par quatre bourgs importants. C'est dans cette région que les jésuites ont élevé en 1639 leur résidence de Sainte-Marie.

Les Attignaouantans, dont ils étaient une partie, étaient la nation huronne la plus nombreuse et apparemment la plus ancienne de la ligue. Les trois autres se trouvaient contenues en six bourgs: un seul pour les Tohontaenrats, deux pour les Attingnenongnahacs et trois pour les Ahrendaronons. Mais les Attignaouantans habitaient en dix-sept bourgs ou villages. Ils étaient la nation la plus influente, formant à peu près la moitié de la ligue, soit environ cinq mille âmes après les épidémies de 1635-1639. Les trois autres habitaient l'arrière-pays, remontant de petites rivières et occupant des lacs, tandis que les Attignaouantans accaparaient tout le littoral de la baie Georgienne, une longueur de quelque 108 kilomètres de rivage. C'était une situation privilégiée pour un peuple dont l'occupation masculine principale était la pêche. Cet avantage du site indique qu'ils étaient les plus anciens habitants du pays. Et cela est corroboré par le nombre de leurs agglomérations, dix-sept, dont la plupart étaient de simples hameaux sans fortifications. Cela faisait contraste avec le petit nombre des bourgs puissamment fortifiés des autres nations huronnes, et aussi avec la forme ordinaire des habitations iroquiennes. C'était, croyons-nous, l'effet d'une habitude très ancienne de la sécurité dans un pays retiré.

Des quatre nations huronnes, les Attignaouantas sont celle sur laquelle nous possédons les renseignements les plus abondants. De 1634 à 1639, époque où les missionnaires furent les plus attentifs aux détails ethnographiques, ils n'ont guère sorti de ses limites, ayant pris d'abord logis à Ihonatiria, petit village situé au coeur du pays. La résidence centrale de Sainte-Marie fut aussi bâtie dans cette nation, qui fut le plus profondément entamée par la prédication chrétienne. Les Attignaouantans restèrent fidèles aux missionnaires, les suivant à Québec en 1650 et refusant en 1657 de les quitter malgré la pression des Agniers et des Onontagués. C'était aussi chez les Attignaouantans que Sagard avait vécu en 1623-1624. Ils étaient si étendus, dans leur pays d'origine, qu'on peut les partager en trois régions ou provinces, possédant un conseil particulier présidé par un capitaine principal. La première était formée par les bourgs avoisinant Ossossané, chef-lieu de la nation, situé à sa limite sud-ouest sur le sentier partant vers les Pétuns et les Neutres. C'était aussi la voie d'invasion des Tsonnontouans. Ainsi, Ossossané était le boulevard dominant la plus ancienne route du commerce et protégeant la ligue entière de ce côté. La seconde province était appelée la Pointe par les missionnaires. C'était la presqu'île où est aujourd'hui 
situé Lafontaine; Ihonatiria s'y trouvait. Elle contenait le plus grand nombre de villages, mais petits et sans défenses. Elle avait son propre conseil, et un capitaine y faisait figure de chef principal. Les ataronchronons habitaient la troisième province, à l'est d'une région marécageuse drainée par la rivière Wye. Leurs quatre bourgs assez nombreux et importants contenaient quelques 1400 âmes. Et l'un des capitaines y tenait aussi la vedette. Le bourg le plus nombreux de toute la Huronnie n'était pas chez les Attignaouantans. C'était Téanaustaié, chef-lieu des Attingnenongnahacs. Mais Ossossané; de moitié moins populeux, était tenu pour le coeur du pays.

Deux incidents intimement reliés feront sentir mieux que toute autre considération à quel point était vive dans une nation iroquienne la conscience de l'unité: la fête des morts de 1636 et le schisme d'Aénons, tous deux arrivés dans la nation des Attignaouantans. La dernière fête des morts avait eu lieu en 1624, Sagard y assistant et en ayant laissé le récit. Elle revenait en 1636, le $P$. de Brébeuf et ses confrères s'y trouvant pour raconter. Entre temps, les morts avaient été enterrés dans les cimetières particuliers des villages. Ils y étaient placés, tout enveloppés de leurs peaux de castor et de leurs caisses d'écorce, sur des échafauds élevés de terre. Les morts à la guerre avaient cependant été enterrés. Les noyés, qui l'étaient aussi, avaient été décharnés avant d'être mis en terre et leurs chairs avaient été brûlées. Les petits enfants, eux, avaient été enterrés sur les chemins, pour rentrer, croyait-on, dans le ventre des femmes qui passaient. Ces sépultures étaient temporaires. Elles allaient toutes être relevées et portées dans une même fosse de toute la nation à l'occasion de la fête des morts.

Au temps de préparer la fête, la Pointe, amas de villages pauvres et négligés, prenait conscience de deux importants avantages qu'elle venait d'acquérir: le fait d'être devenue le débarcadère le plus accessible pour les marchandises européennes, spécialement le fer, et celui de posséder chez elle les jésuites, gages de l'alliance française. Cela fit naître une grande ambition chez quelques capitaines, celle de réunir les villages en un grand bourg bien fortifié. Aénons, capitaine d'Ouenrio, prit la tête du mouvement. Mais plusieurs villages, dont Ihonatiria, hésitèrent à le suivre. Ce projet n'était pas pour plaire aux chefs d'Ossossané, auxquels le nouveau bourg, mieux situé, enlèverait la vedette. Aénons s'efforçait d'attirer les jésuites chez lui; Ossossané les assiégeait d'invitations à s'y transporter. Un jour, Aénons vint trouver Brébeuf, l'invitant au nom du conseil de la nation à faire enterrer deux Français, Guillaume Chaudron et Étienne Brûlé, dans 
l'unique fosse des Attignaouantans. Chaudron reposait à Ossossané; Brûlé, à la Pointe. Après avoir hésité, le supérieur accepta, à condition d'enterrer les Français à part avec les Hurons baptisés et déjà mort et de planter une croix sur leurs tombes. Le conseil accepta. C'était une occasion unique d'ouvrir un cimetière chrétien, qu'on n'avait pu faire jusque là et qui en réalité ne sera pas commencé avant plusieurs années.

En. effet, lors d'une nouvelle réunion du conseil, la dissension éclata entre les parties rivales. Les cinq villages qui suivaient Aénons résolurent de faire leur fête et leur fosse à part, pendant que le reste de la nation prenait fait et cause pour Ossossané. Aénons réclama le corps de Brûlé, mais refusa celui de Chaudron. Les jésuites décidèrent de ne pas troubler leurs sépultures. Ils assistèrent toutefois avec leur village d'Ihonatiria à la grande fête d'Ossossané, laissant les dissidents à leur fête particulière. Les prodiges de diplomatie, d'adresse et de patience déployés alors par les chefs pour réduire la division en démontrèrent la gravité. La division de la "chaudière", c'est-à-dire du festin des morts, était la division de la nation elle-même. La nation, ici, n'est pas la ligue huronne, mais les seuls Attignaouantans, les autres nations n'assistant qu'à titre d'invitées. Nulle part autant que dans cet incident, il n'apparaît que la nation, et non la ligue, est l'unité socio-politique chez les Hurons. Malgré les efforts déployés, le schisme subsista quelque temps. Les villages séparés ne réussirent pas à se former en un seul bourg. Aénons mourut en 1637 durant un voyage à Trois-Rivières. Les jésuites, après avoir ouvert une résidence à Ossossané en 1637, transportèrent celle d'Ihonatiria à Téanaustaié, chef-lieu de la nation voisine, en juin 1638. Et les maladies ravagèrent les villages de la Pointe.

Il faut descendre au niveau du bourg ou du village pour trouver l'assise des fonctions de gouvernement. Ce n'est pas qu'il n'y ait quelques personnages, uniformément appelés chefs ou capitaines, dont le service intéresse toute la nation. Mais leur fonction est limitée à un secteur restreint de l'activité commune. Il y a un grand chef de guerre pour toute la nation, nommé Atsan en 1637, dont relèvent les affaires militaires. Un autre est chef de la navigation, qu'on n'entreprend pas sans son aveu. Un troisième est maître des routes de commerce tandis que le grand chef des Attignaouantans se réserve la nouvelle route ouverte vers les Français. Les officiers de la communauté sont en premier lieu attachés au service des bourgs et villages. Chaque agglomération a d'abord deux chefs: l'un qui est dit de conseil, l'autre de guerre. Celui-ci n'intervenant qu'en matière militaire, les nombreuses affaires de la communauté retombent sur 
les épaules du premier, qui demeure le plus important et qui doit déployer le plus de sagacité politique. Mais ces deux ne sont pas les seuls chefs. Chaque fraction de nation adoptée résidant dans un bourg a aussi une direction semblable: un chef de conseil et un chef de guerre. Ainsi, dans un bourg comme Scanonaenrat, unique habitation des Tohontaenrats, on pourra compter jusqu'à douze capitaines. Chacun se confine dans son office et son service, se défendant d'intervenir en matière étrangère à sa compétence. Le capitaine de conseil du bourg principal porte naturellement la voix de toute la nation aux assemblées de la ligue ou devant les étrangers. Mais la puissance d'un capitaine n'est ni impérative ni coercitive; sa fonction est un service d'animation et d'exécution, honorable certes, mais très onéreux et peu profitable. C'est pourquoi il faut avoir des aptitudes spéciales pour le remplir et plusieurs s'en excusent quand l'honneur est offert. Personne aussi ne devient capitaine que par la reconnaissance générale. Il n'y a guère d'autre hiérarchie entre les capitaines que celle du dévouement au public et du talent déployé dans l'exercice de la charge.

Il n'y a presque pas de domaine où l'intervention d'un ou de plusieurs capitaines n'est requise, exception faite des rapports entre membres d'une famille particulière, celle-ci étant considérée comme souveraine dans sa régie interne. Tout ce qui requiert l'assistance de la communauté, construction d'une cabane ou rite guérisseur d'une maladie, est occasion d'un recours aux capitaines, qui à leur tour mettent en branle les ressorts de la communauté, d'un village ou même de plusieurs.

Aussi les capitaines, même collectivement, ne forment-ils pas l'instance suprême de gouvernement. Ils sont des agents d'exécution. La puissance de la communauté, analogue aux pouvoirs législatif et judiciaire d'un âge plus évolué, réside en définitive dans le conseil. Les capitaines, du moins les principaux, sont ceux qui convoquent les conseils publics, qui y exposent les questions mises en discussion, qui résument les propos entendus et formulent les conclusions. C'est là que le talent et le prestige des meilleurs capitaines se font spécialement valoir. Mais les membres attitrés des conseils sont proprement les anciens. La qualité d'ancien n'a rien de négligeable. Si elle requiert l'âge, il ne s'ensuit pas que tous les vieillards la possèdent. Elle est une qualité publiquement reconnue à certains membres de familles distinguées par leurs services à la communauté entière. Il y en a dans tous les villages. Ce sont eux qui opinent et leurs décisions sont mises en oeuvre par les capitaines. Ces résolutions sont contraignantes pour la communauté entière. Il y a des conseils à tous les 
niveaux de la composition sociale. Même les cabanes et les familles ont les leurs, auxquels participent les femmes comme les hommes; mais ce ne sont pas des conseils publics. Ceux-ci, réunis et présidés par un capitaine, se font au niveau du village, ou de deux ou trois villages impliqués dans le même problème, ou encore d'une région comme la Pointe et les Ataronchronons, ou bien, au sommet, de la nation entière. Il y a enfin des conseils de ligue, où sont agitées les questions intéressant plusieurs nations, le plus souvent de défensive ou d'offensive militaires. Les votes se prennent par groupes représentés, familles, villages ou nations, qui donnent chacun une voix commune et unanime. $\mathrm{La}$ résolution ne s'impose que si le scrutin global est unanime. La dissidence libère de l'obligation de l'exécuter. Le désaccord ou l'absence d'un seul groupe peut faire manquer un conseil ou forcer de l'ajourner. Les fonctions de capitaines et la participation aux conseils publics étaient exclusivement masculines au temps de la mission huronne. Sagard affirme carrément l'exclusion des femmes. Les Relations le confirment en indiquant que ces dernières, même celles de la cabane où se tenait le conseil, après avoir préparé la salle et les feux nécessaires à ces assises, devaient s'en retirer avant l'ouverture de la séance et en rester éloignées aussi longtemps qu'elle durait. Les missionnaires français de ce temps n'auraient pu manquer, au cours de seize années, de noter une participation féminine directe aux conseils des Hurons. Mais ils ne le font pas. On sait toutefois qu'à la même époque, on comptait des femmes parmi les oïanders, terme iroquois qui est équivalent à celui d'anciens chez les Hurons. Elles avaient rang, crédit et parole comme les hommes. En outre, le P. Pierre Potier, missionnaire du village huron voisin de Détroit en 1747, y mentionne des anciennes à peu près en même nombre que les anciens, participants aux conseils. Il me semble donc que ces anciennes ont été introduites après la destruction et l'exil des Hurons, peut-être à l'exemple des Iroquois. Car à Lorette, après 1673, même s'il se trouve des Iroquoises ayant eu la dignité d'oïanders dans leur pays, on ne voit pas de participation féminine aux mêmes conseils.

Les institutions socio-politiques des Hurons ont leurs racines dans la famille biologique. Quoi qu'en ait autrefois pensé Jean-Jacques Rousseau, la société humaine n'est pas l'effet d'un contrat négocié ou d'une constitution raisonnée. Elle est l'aménagement humain, c'està-dire conscient et créateur, d'une famille qui s'accroît naturellement et invente des formes de conservation adaptées à ses besoins. Le modèle ultime est la nature, et la nature vivante. On constate dans les sociétés iroquiennes un effort, favorisé par la stabilité de l'habitat découlant de l'agriculture, pour conserver le plus longtemps possible 
ensemble les membres d'une même famille. Cela procure la physionomie spéciale de la cabane, longue tonnelle d'écorces ponctuée sur son axe de feux à intervalles réguliers, de part et d'autre desquels prennent place les couples avec leurs enfants. La solidarité de la famille est exigence d'une économie encore primaire; elle est la garantie la plus fondamentale de la sécurité des invididus. Mais elle ne peut résister à l'énergie centrifuge de la croissance démographique. C'est de là que jaillissent des aménagements sociaux variables, que l'homme fait à sa manière, consciente et construite.

Les cabanes huronnes étaient les cellules d'où rayonnait le dynamisme social. Les sources en mentionnent de douze feux, ayant une capacité de vingt-quatre ménages. Mais leur grandeur était très variable. On en trouvait aussi d'un seul ménage. La moyenne paraît avoir été de cinq ménages. En principe, on y trouvait réunies les générations vivantes d'une même souche: grands-parents, leurs enfants et leurs petits-enfants. On fait souvent grand état d'une habitation qui aurait été matrilinéaire. Cela signifie que les filles d'une famille auraient demeuré dans la cabane de leur naissance en se mariant, y entraînant leur époux. Ainsi, les garçons mariés auraient habituellement quitté la cabane de leur père, tandis que les filles y seraient restées avec leurs enfants. Une étude attentive des sources nous révèle que cette pratique n'est pas observable. Il y a autant de fils mariés que de filles demeurant dans la cabane de leur naissance, et même davantage. Il arrivait donc souvent, et même le plus souvent, que les filles quittaient leur mère pour vivre avec leur époux. Un témoignage de Joseph Chiouatenhoua présente cet arrangement comme ordinaire. Et il faut tenir compte des autres cas, où mari et femme ont leur cabane particulière, qui n'est celle d'aucun parent. Il n'y a donc pas de constance dans la pratique matrilinéaire. Ce qui est vrai, c'est que l'époux contracte en se mariant l'obligation d'assister la cabane de sa femme; mais l'épouse en assume une réciproque envers la cabane du mari. En réalité, ces engagements demeurent souvent symboliques.

On dit aussi que les héritages étaient transmis en ligne féminine. Il est difficile d'expliciter ce que signifierait cette loi. Mais on doit faire quelques remarques. Un mort ne laissait guère d'héritage chez les Hurons. Ce qu'il possédait en propre était ce qu'il avait fabriqué de ses mains et à son usage, ou ce qui tenait à son corps. Cela était en grande partie enseveli avec lui. Il en emportait souvent plus en présents qu'on lui faisait. Ce qui restait était distribué par gratitude pour les condoléances. Mais ce qui était à l'usage de son ménage, provisions ou autres choses, restait à ce dernier, sans formalités. 
Il laissait cependant parfois d'autres biens plus précieux; une dignité de capitaine ou d'ancien, un privilège acquis à la famille et dont il était le régisseur. C'était là un patrimoine de la cabane, qui ne souffrait pas de le voir sortir d'elle-même. Il y avait donc matière à succession. Cette succession était hériditaire, c'est-à-dire qu'elle passait de génération en génération, dans la même lignée. Mais toute la communauté sociale avait intérêt à ces biens. Toute succession eût été vaine, qui n'eût été publiquement agréée par les organes de la communauté, le conseil du village ou de la nation.

Champlain a écrit que cette transmission se faisait du défunt à un neveu par les soeurs et non pas aux enfants, par crainte de l'illégitimité de ces derniers. On est assez gêné devant une institution sociale fondée sur la jalousie des maris. Mais Sagard corrige Champlain en mettant les fils en première ligne de succession. Cela convient mieux à la pratique qu'on voit observée. Naturellement, donc, la succession va d'abord au fils, que l'avantage de sa naissance a déjà invité à vivre dans la cabane de son père avec sa famille, pour prendre sa place en temps voulu. La transmission est alors si fortement justifiée que le successeur n'a pas besoin de prendre le nom de son prédécesseur. Il y a en effet de telles successions sans transmission du nom. Anenkhiondic, premier chef de la nation des Attignaouantans, décédé en 1638, continuait au temps de Brébeuf le même office qu'Aouindaion tenait en 1624, au temps de Sagard. S'il n'y a pas de fils survivant, ou qu'il n'ait pas les qualités qui le feront accepter publiquement, ou qu'il refuse le fardeau comme il peut le faire, le choix se reporte sur le consanquin le plus rapproché, d'abord parmi les frères. Atironta, premier chef de guerre des Ahrendaronons, eut ainsi pour successeur son frère, Aéoptahon, auquel fut solennellement conféré son nom d'Atironta. Cette résurrection du nom, comme on l'appelait, conférait au nouveau dignitaire toutes les attributions et les responsabilités du précécesseur, en même temps que son poste dans la cabane, à l'exception des droits conjugaux. À défaut d'un frère, les neveux venaient ensuite en ligne. Mais d'abord les neveux par les soeurs du défunt. Pourquoi les soeurs? La raison la plus plausible nous paraît être que, dans l'opinion des Hurons, les enfants étaient plus prochainement liés par le sang à leur mère qu'à leur père. Les neveux par les femmes étaient ainsi plus proches parents du défunt que leurs cousins issus de ses frères. Dans ce cas aussi, il y avait transmission du nom. Cette formalité, à la suite de ce qui vient d'être dit, semble avoir compensé ou le saut d'un degré de parenté qu'il a fallu faire ou la nécessité de prendre le successeur hors de la cabane de l'ancien chef. 
Il faut souligner que, la responsabilité à transmettre étant un patrimoine de la cabane, c'est aussi toute la cabane, assistée d'ailleurs par tous les proches parents vivant à l'extérieur, qui fait le choix du successeur. Les femmes comme les hommes participent à ce choix, mais aucune de nos sources ne réserve le choix aux femmes seules. Mieux encore, la veuve du défunt n'y joue qu'un rôle passif. Ce sont les parents de feu Atironta qui choisissent Aéoptahon pour lui succéder. Or celui-ci a été baptisé et l'on sait que la veuve du prédécesseur est tout à fait animée contre les chrétiens. La résurrection des noms était une pratique générale aussi bien des Algonquiens que des Iroquiens et elle avait partout les mêmes effets. Elle n'était d'ailleurs pas réservée aux officiers publics, mais elle avait lieu également pour les particuliers. Ainsi, à Sillery, le nom d'un mari défunt, qui laissait une veuve et des orphelins, fut donné par la famille à un frère, afin qu'il prît charge de la cabane. Tout cela s'est fait sans participation de la veuve. On ne peut donc justifier par là une succession féminine et matrilinéaire, déjà en soi fort difficile à concevoir. Le lien du sang entre les neveux et le défunt, même s'il passe par les soeurs du feu capitaine, est toujours celui de la lignée masculine. Les soeurs du mort ne doivent rien à sa veuve et celle-ci n'a rien à leur transmettre qui soit de sa propre lignée. Car le défunt a succédé lui-même à son père ou à son grand-père.

Ces possessions prisées de la cabane, que sont les charges de capitaine et d'anciens, les privilèges commerciaux et autres à retentissement public, ne sauraient être laissées entièrement à sa disposition. Les organismes communautaires, ceux du village ou ceux de la nation, laissent volontiers à la famille le soin de désigner le titulaire. Mais cette désignation serait sans effet, si elle n'était ratifiée et agréée par les instances publiques. Il y a même des cas où, le capitaine défunt ayant mal servi sa communauté, les conseils prenaient sur eux d'instituer son successeur. De tout cela, la famille tenait compte en procédant à l'élection. Puis, par l'intermédiaire de quelques capitaines, l'élu était présenté au conseil du village ou à celui de la nation, avec l'accompagnement nécessaire des présents et des festins. Le conseil entérinait l'élection et recevait l'élu dans l'exercice de sa fonction. Les anciens avaient besoin d'une confirmation moins éclatante que les capitaines. Leur rôle, pour être à certains égards plus décisif, était beaucoup moins actif, individuel et visible. Ils étaient davantage liés au lieu qu'ils habitaient. Aussi n'exigeaient-ils pas une publication aussi étendue que les capitaines. 
N'ayant pas en vue d'épuiser en ce court exposé tous les aspects de la société huronne, je ne puis cependant terminer sans avouer une difficulté lancinante qui m'a tracassé tout au long de sa rédaction. Les traits que j'ai perçus de la société huronne me mettent en désaccord avec un grand confrère et prédécesseur auquel je n'oserais pas me comparer, le P. Joseph-François Lafitau (1681-1746) ${ }^{6}$. Le P. La Lafitau ne manquait pas d'autorité. D'une très vaste érudition classique, d'une vigoureuse et créatrice intelligence, il est encore un témoin historique de première main en ce qui concerne la culture iroquoise, qui avait tant de points communs avec la culture huronne. Il est très difficile de faire ombre à un aussi grand personnage. Je n'ai pour excuse que ce fait: le P. Lafitau n'a connu les Hurons que dans les mêmes livres que moi. Dans ce champ réservé, me serait-il permis de confronter ma compréhension des témoignages avec la sienne?

Le P. Lafitau est celui qui a formé le mot "gynécocratie ou empire des femmes " pour désigner l'état socio-politique à la fois des Iroquois et des Hurons. Il dérive ce caractère d'une comparaison avec les Lyciens de l'antiquité, qui avec de nombreux autres peuples auraient vécu d'un pareil régime. Ce dernier représenterait, à son dire, un stade de l'évolution des sociétés. Mais je n'ai pu me rendre compte clairement de l'ensemble des éléments qui, chez les Iroquois et les Hurons, manifesterait ce caractère gynécocratique. Ce qui reste certain est que ces affirmations insistantes à cet égard ont été généralement reçues, qu'on a brodé de diverses manières sur ce trait et qu'il est le plus ordinairement accepté, dans la langue d'aujourd'hui, d'appeler les Hurons et les Iroquois des sociétés matriarcales. Je ne parlerai ici que des Hurons, qui sont proprement mon sujet.

On ne déniera pas aux femmes leur importance sociale en tout temps, préhistorique ou historique. Il est certain que la matrone, comme le P. Lafitau l'appelle, femme, ou mère, ou fille du chef de cabane qui est toujours un homme dans les Relations huronnes - possède une pleine autorité sur le fonctionnement interne de la cabane. L'homme ne l'entrave pas et lui fait confiance, représentant lui-même la cabane au dehors. Cette initiative de la maîtresse huronne est égale à celle de la femme du chasseur principal chez les Algonquins, à cela près que la maisonnée huronne est d'ordinaire plus nombreuse. L'importance des femmes huronnes est encore accrue du fait qu'elles procurent en culti-

\footnotetext{
6 [ François-Joseph] Lafitau, Moeurs des sauvages amériquains comparées aux moeurs des premiers temps, 4 vol., Paris, Saugrain et Hochereau, 1724.
} 
vant la plus grande partie des aliments. Mais cet aspect économique n'a que peu de considération dans cette culture. L'homme lui-même estime ses occupations à plus haut prix. Il n'appartient pas à la matrone de décider une expédition pour venger la perte d'un membre de la cabane, bien qu'elle puisse en avoir la première idée. C'est par la délibération de toute la cabane, hommes et femmes, que le projet prend forme. Porté de là à un capitaine de guerre, normalement évalué par le conseil, il sera enclenché. Nous n'avons pas trouvé chez les Hurons les conseils de femmes dont parle le P. Lafitau, où s'esquissent des plans politiques à adopter par les conseils. Ils ne pourraient être que privés et les conseils de cabane ont déjà un rôle en ce sens pour les problèmes particuliers de la famille. Il est vrai que les membres de sa cabane sont les premiers conseillers d'un capitaine ou d'un ancien, les femmes parmi les autres. Cela ne donne pas à celles-ci un pouvoir sur la société.

La nation, chez les Hurons, est une lignée se réclamant d'un ancêtre masculin. Elle se définit par la succession des générations masculines. Les capitaines sont exclusivement des hommes. Au temps de Sagard et de la mission huronne, les femmes non seulement ne siégeaient pas aux conseils publics, mais elles n'avaient même pas la permission d'y être présentes, alors qu'on exhortait les jeunes hommes à y assister. Les femmes prenaient part à quelques festins publics, mais d'autres leur étaient interdits. Elle exerçaient, certes, leur part d'influence dans les conseils familiaux, mais conjointement avec les hommes. Comme on l'a vu plus haut, la succession aux charges publiques, ordinairement héréditaire, était masculine, au moins du grand-père au petit-fils, sinon au fils. Il n'y aurait aucun sens à chercher une succession de grand-mère à fille et à petite-fille. Les mâles hurons méprisaient pour eux-mêmes les occupations féminines. Les petits garçons refusaient les services qu'on demandait aux petites filles. C'était faire injure à un homme que de lui imputer une conduite ou des émotions féminines. Le P. Lafitau, qui n'ignorait pas cette prévalence sociale des hommes, l'expliquait par une procuration donnée par les femmes aux hommes d'exercer un pouvoir proprement féminin. L'hypothèse est non seulement invérifiable, mais elle embrouille les faits sociaux attestés, plutôt qu'elle ne les explique.

Nous sommes, avec les Iroquois et les Hurons, en présence de sociétés néolithiques. La méthode comparative, brillamment appliquée par' le P. Lafitau, est excellente, mais à condition d'opérer selon une économie convenable. Les populations autochtones d'Amérique ont été séparées du vieux continent lorsque celui-ci n'était encore l'habitat que 
de bandes chasseresses et nomades, le conquérant pièce par pièce. Depuis lors, les envahisseurs de l'Amérique ont fait une expérience isolée et séparée. Les peuples euro-asiatiques parmi lesquels le P. Lafitau cherche aux Hurons-Iroquois des ancêtres et une genèse ont été tous inscrits déjà au rôle de l'histoire et de la mémoire humaines. Mais les Hurons et les Iroquois, encore à notre dix-septième siècle, étaient enveloppés dans l'oubli et les fables de l'enfance. C'est par un accident fortuit et heureux qu'ils furent soudain révélés dans leur état natif. Ils sont aptes à nous en apprendre davantage sur l'enfance oubliée des Euro-Asiatiques que ne le feraient ces derniers, déjà à l'aurore de l'histoire, sur les Hurons-Iroquois. Une étude attentive et pénétrante des autochtones d'Amérique en révèle plus sur les origines du vieux continent humain que celui-ci ne peut en expliquer sur eux. Et le monde indigène américain ne fournit pas d'appui à la tradition des Amazones, dont l'antiquité euro-asiatique a fait si grand état.

Lucien CAMPEAU, S.J. 